\title{
Elbow dislocation with bicondylar humeral fracture in an adult: a unique type of injury
}

\author{
Mohit Kumar Arora ${ }^{1 *}$, Ela Madaan², Sandeep Kumar ${ }^{3}$ \\ 'Department of Medicine, GTB Hospital, New Delhi, India \\ ${ }^{2}$ Department of Medicine, Delhi Heart Hospital, New Delhi, India \\ ${ }^{3}$ Department of Medicine, SDN Hospital, New Delhi, India
}

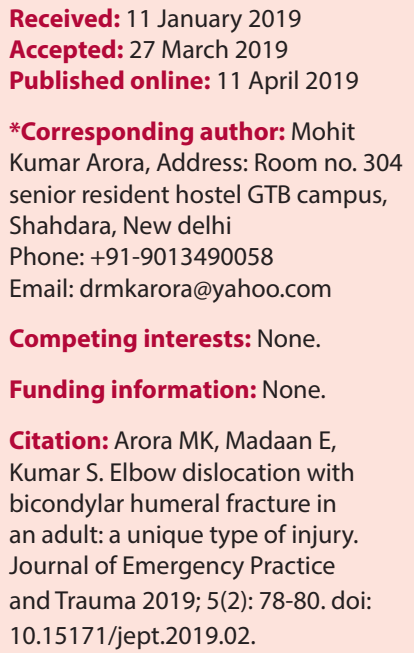

\begin{abstract}
Simultaneous fracture of lateral condyle and medial epicondyle of humerus along with elbow dislocation is very rare injury in adults. Only a few cases have been reported in literature in pediatric age groups. The authors describe a case report of fracture of lateral condyle and medial epicondyle of humerus along with elbow dislocation in a young adult. The patient sustained injury in the form of fall from bike. Clinically the patient had swelling and deformity of the elbow joint. There were contusions present in the skin around the elbow joint. There was no distal neuro-vascular deficit. Appropriate radiological investigations were done. The elbow joint was then reduced and found to be unstable. Hence, patient was taken up for surgery in the form of open reduction and internal fixation. The functional outcome of the surgery is presented in the case report. Open reduction and internal fixation are the treatment of choices in these types of cases.

Keywords: Lateral condyle, Elbow, Dislocation
\end{abstract}

\section{Introduction}

The association of lateral condyle and medial epicondyle fracture of humerus along with elbow dislocation in a young adult is a rare injury. Literature search shows that this combination of injuries has been described in pediatric age group $(1,2)$. We present a case report of elbow dislocation along with lateral condyle and medial epicondyle fracture.

\section{Case Presentation}

A 25-year-old male presented to the emergency department of our institution with pain, swelling and deformity of the left elbow. There was a history of fall from the bike. There was no history of loss of consciousness, seizure, vomiting and ENT (ear, nose and throat) bleed. Clinically the elbow was dislocated and contusions were present over skin around the elbow joint. Radiographs (Figure 1) showed elbow dislocation along with the fracture of lateral condyle and medial epicondyle. CT scan (Figure 2) was done to confirm the findings. Elbow dislocation was reduced and splintage was given. However, elbow joint was found to be unstable post reduction. The patient was taken up for surgery where lateral condyle was fixed with a $4 \mathrm{~mm}$ cannulated cancellous screw (CCS) and medial epicondyle was fixed with $\mathrm{k}$ wire (Figure 3). Post-operative period was uneventful. The patient was immobilized for 3 weeks in a cast and gradually the elbow joint range of motion exercises was started. The $\mathrm{k}$ wire was removed at 6 weeks post operation. At the last follow up (Figures 4-6), the patient was asymptomatic, pain free and had gained
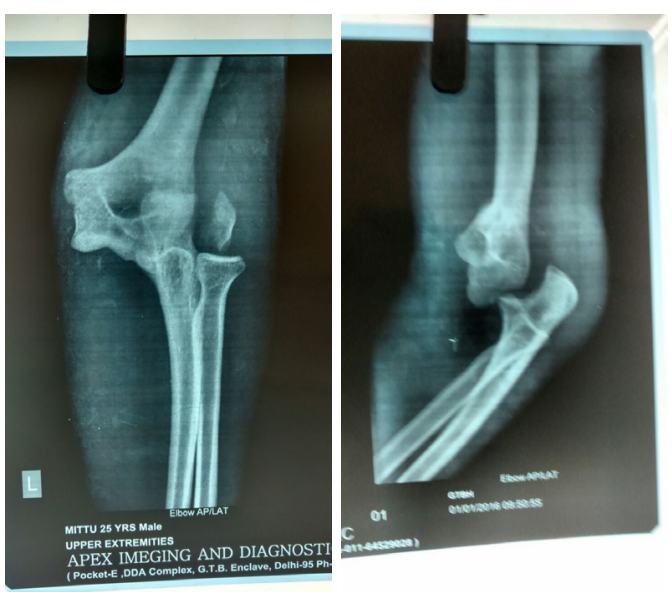

Figure 1. $X$ rays (AP and Lat views) showing elbow dislocation along with fracture lateral condyle. Fracture medial epicondyle cannot be visualized here. 


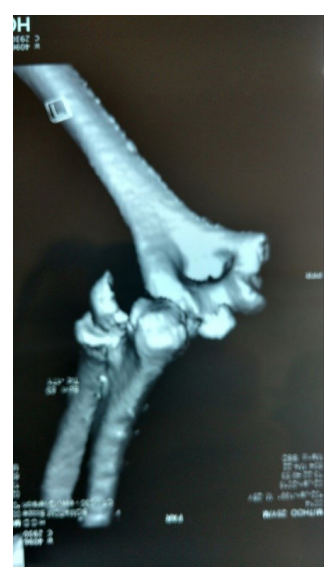

Figure 2. 3D CT reconstruction. Here both fracture fragments can be visualized.

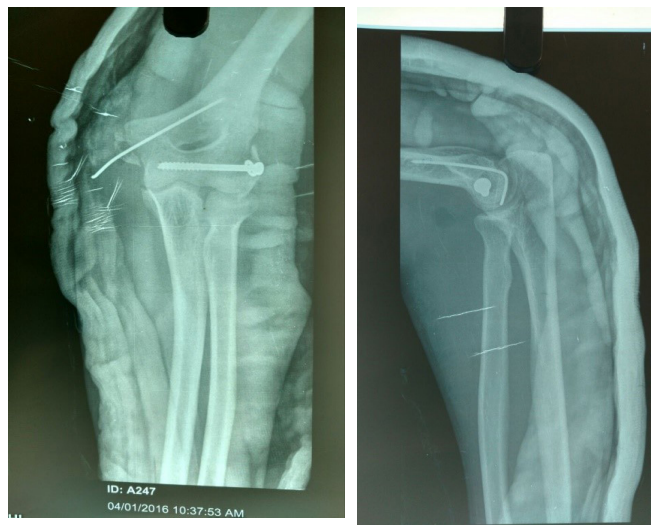

Figure 3. Post op X-rays (AP and Lat views) showing fixation of lateral condyle with a $4 \mathrm{~mm} \mathrm{CCS}$ and medial epicondyle with $\mathrm{k}$ wire.
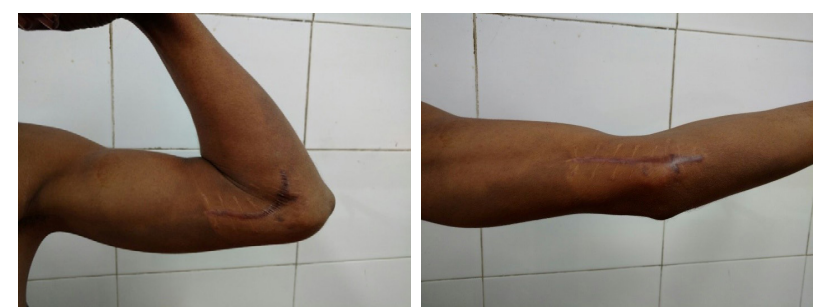

Figure 4. Flexion and extension at the final follow up.

almost a full range of motion of the elbow joint.

\section{Discussion}

The elbow dislocation rate is $6-13$ cases per 100,000 people and accounts for $11 \%-28 \%$ of all injuries to the elbow (3). Elbow joint stability is provided by static factors which include congruent joint surfaces, capsule and ligaments around the joint, while the dynamic factors include muscles around elbow $(4,5)$. The primary stabilizers of the elbow joint are collateral ligaments (medial and lateral) and the secondary stabilizers include radial head, common flexor and extensor tendons origin and the capsule. However, its association with lateral condyle fracture and medial epicondyle is very rare. The concomitant fracture

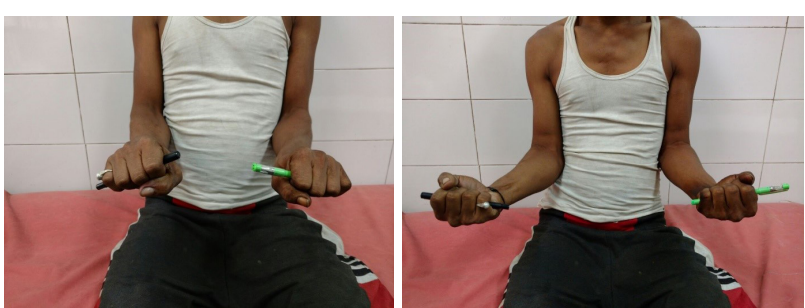

Figure 5. Pronation and supination movements at the final follow up.

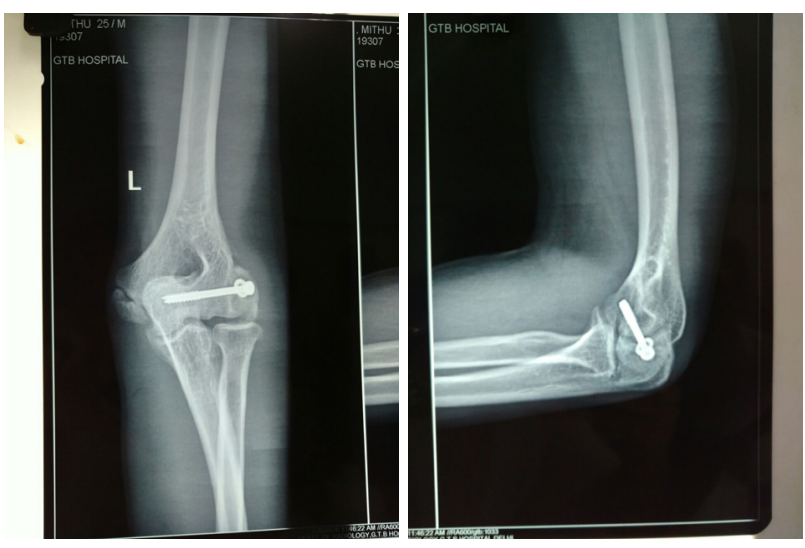

Figure 6. X-rays (AP and Lat views) at final follow up.

of both lateral condyle and medial epicondyle makes the elbow joint unstable as in our case. The sequence of events leading to this type of injury can be summarized as follows. The patient had fallen on an outstretched hand with elbow in full extension and forearm in pronation. Associated valgus force at the elbow would have led to avulsion of medial epicondyle along with impaction of radial head over capitellum leading to the fracture of lateral condyle. In such cases, open reduction and internal fixation is the treatment of choice. We used both medial and lateral approaches around the elbow. The rarity of this type of injury necessitates appropriate management to ensure optimal functional outcome. There is no substitute for thorough clinico-radiological examination.

\section{Conclusion}

The combination of lateral condyle and medial epicondyle fracture humerus along with elbow dislocation is very rare especially in adults. Proper radiographs supplemented by CT scan and thorough clinical examination are required to diagnose these type of injuries. These cases should always be treated by open reduction and internal fixation.

\section{Authors Contribution}

MKA did the surgery, while EM and KS prepared the manuscript along with MKA.

\section{Ethical issues}

The patient's consent for publication of his case was obtained prior to the submission of this manuscript. 


\section{References}

1. Gani NU, Rather AQ, Mir BA, Halwai MA, Wani MM. Humeral biepicondylar fracture dislocation in a child: A case report and review of the literature. Cases J 2008; 1(1): 163. doi: 10.1186/1757-1626-1-163.

2. Taylor GR, Gent E, Clarke NM. Biepicondylar fracture dislocation of a child's elbow. Injury 1997; 28(1): 71-2. doi: 10.1016/s0020-1383(96)00138-6.
3. Hobgood ER, Khan SO, Field LD. Acute dislocations of the adult elbow. Hand Clin 2008; 24(1): 1-7. doi: 10.1016/j. hcl.2007.11.012.

4. Bryce CD, Armstrong AD. Anatomy and biomechanics of the elbow. Orthop Clin North Am 2008; 39(2): 141-54. doi: 10.1016/j.ocl.2007.12.001.

5. Lockard M. Clinical biomechanics of the elbow. J Hand Ther 2006; 19(2): 72-80. doi: 10.1197/j.jht.2006.02.004. 